\title{
Comparative Study of Diverse Face Recognition Approaches along with Intrinsic Worth and Recognition Rate
}

\author{
Shivang Shukla \\ Department of Computer Science \\ Medi-Caps Institute of Science and Technology \\ Indore, Madhya Pradesh, India
}

\author{
Sourabh Dave \\ Department of Information Technology \\ Medi-Caps Inst. Of Science and Technology \\ Indore, Madhya Pradesh, India
}

\begin{abstract}
Face recognition is one of the research areas which have always attracted the attention of the researchers and research community. Because it is a varied application in automation, authentication, medical diagnosis, access control, surveillance and security applications. Face recognition is one of the most successful applications of image analysis and understanding. It has gained much attention in recent years. Various algorithms were proposed and their own have some limitations and merits for end-user application. The aim of this paper is to select an optimum technique of face feature selection. That can be used for multiple face object detection because feature representation and classification are two key steps for face recognition systems. Therefore, in this paper, a comparison of six effective feature computation methods is performed. In this comparison PCA, LDA, and ICA, SIFT, SURF, and ORB are considered. After comparison of these features, a promising feature extraction technique is selected for further application development.
\end{abstract}

\section{General Terms}

Pattern Recognition, Principal Component Analysis, Machine Learning Application, Karhunen- Loeve Transform, SpeededUp Robust Feature, Haar wavelets

\section{Keywords}

Face Recognition, Feature Extraction, PCA, ICA, LDA, ORB, SIFT, SURF

\section{INTRODUCTION}

It is broadly recognized that the face acknowledgment has assumed an essential part in reconnaissance framework as it needn't bother with the protest's collaboration. The real focal points of face-based recognizable proof over different biometrics are uniqueness and acknowledgment. Face recognition is a biometric system utilized for reconnaissance purposes, for example, the scan for needed offenders, suspected fear mongers, and missing youngsters. The term confront acknowledgment alludes to recognizing, by computational calculations, an obscure face picture. This operation should be possible by contrasting the obscure face and the appearances put away in the database. Face recognition has three phases [1]:
a) Face location detection
b) Feature extraction
c) Facial image classification

Face recognition (FR) has risen as a standout amongst the most broadly contemplated examine points that traverse numerous controls, for example, design acknowledgment, flag preparing, and PC vision. This is because of its various essential applications in character confirmation, security gets to control, keen human-PC association, and programmed ordering of picture and video databases [2]. The face is the index of the mind. It is a complex multidimensional structure and needs a decent figuring system for acknowledgment. While utilizing the programmed framework for face acknowledgment, PCs are effectively confounded by changes in enlightenment, variety instances and change in points of appearances. Various procedures are being utilized for security and verification purposes which incorporate regions in criminologist offices and military purpose [3].

Face biometrics, useful for a person's authentication is a simple and non-intrusive method that recognizes the face in the complex multidimensional visual model and develops a computational model for it. A ubiquitous property of human perception is our capacity to recognize diverse faces even when they look really similar and recognize hundreds of different individuals with almost no effort. Automated face recognition is an area of Computer Vision inspired by this ability. Facial recognition systems focus on extracting faces from static images and video sequences and deciding whether they belong to a database of known individuals [4].

The face is the index of the mind. It is a composite multidimensional organization and needs a decent figuring method for acknowledgment. While utilizing the programmed framework for face acknowledgment, PCs are effectively confounded by changes in enlightenment, variety in postures and change in edges of countenances. Various procedures are being utilized for security and verification purposes which incorporate ranges in criminologist offices and military purpose [4].

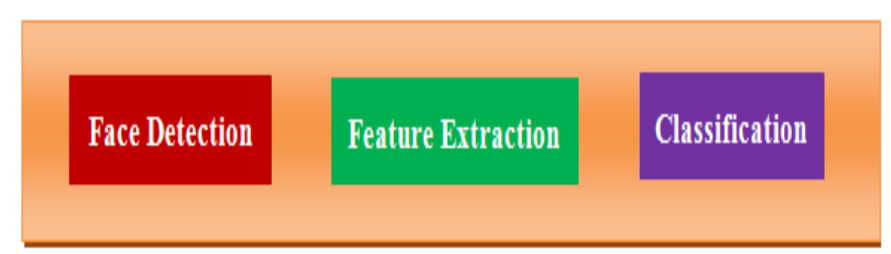

Figure 1: Face Recognition

The face detection and face extraction are carried out simultaneously. The complete process of face recognition can be shown in Figure 1. The initial phase in face acknowledgment framework is to detect the face in an image. The main objective of face detection is to find whether there are any faces in the image or not. If the face is present, then it returns the location of the image and extent of each face. Pre- 
processing is done to remove the noise and reliance on the precise registration.

\section{BACKGROUND STUDY}

Throughout the past few decades, there have been many face location procedures proposed and actualized. A portion of the normal strategies depicted by the analysts of the separate fields are:

\subsection{Principal Component Analysis (PCA)}

PCA is an orthogonal direct change that changes the information to another arrange framework with the end goal that most prominent difference by any projection of the information comes to lie on the rest organize; the second most prominent fluctuation comes up in the second facilitate, et cetera. Eigenfaces [5] otherwise called Principal Components Analysis (PCA) locate the base mean squared blunder direct subspace that maps from the first $\mathrm{N}$-dimensional information space into an M-dimensional element space. By doing this, Eigenfaces (where characteristically $\mathrm{M} \ll \mathrm{N}$ ) accomplish dimensionality reduction by using the $\mathrm{M}$ eigenvectors of the covariance matrix corresponding to the largest eigenvalues. The subsequent premise vectors are gotten by finding the ideal premise vectors that amplify the aggregate change of the anticipated information (i.e. the arrangement of premise vectors that best portray the information). Usually, the mean $x$ is extracted from the data, so that PCA is equivalent to Karhunen-Loeve Transform (KLT).PCA converts a high dimensional data into the low dimensional image in a linear fashion, in which the principal component is not correlated. The recognition rate of PCA-based face recognition outperforms when the number of test images increases, but the rate of recognition decreases of the certain number. PCA reduces the dimension size of an image in a short period of time. There is a high correlation between the training data and the recognition data [6].

\subsection{Linear Discriminant Analysis (LDA)}

LDA is generally used to discover direct blends of components while safeguarding class distinctness. Dissimilar to PCA, LDA tries to demonstrate the contrasts between classes. Great LDA is intended to consider just two classes. In particular, it requires information indicates for various classes be a long way from each other, while focuses on a similar class are close. Subsequently, LDA gets differenced projection vectors for every class. Multi-class LDA calculations which can oversee more than two classes are more utilized [7]. Linear Discriminant Analysis (LDA) is most commonly used as dimensionality reduction technique in the pre-processing step for pattern classification and machine learning applications. The goal is to project a dataset onto a lower-dimensional space with good class-separability in order avoid overfitting ("curse of dimensionality") and also reduce computational costs. The optimal projection (transformation) can be readily computed by applying the Eigen decomposition on the scatter matrices Linear discriminate analysis (LDA) and the related Fisher's linear discriminate are methods used in statistics, pattern recognition and machine learning to find a linear combination of features which characterize or separate two or more classes of objects or events [8] [9]. The resulting combination may be used as a linear classifier or more commonly, for dimensionality reduction before later classification.

\subsection{Independent Component Analysis (ICA)}

Independent component analysis [10] is a technique to find a linear transform for the input data using a basis as statistically independent as possible. Hence, ICA can be considered as a special case of PCA. While applying PCA to an arrangement of face pictures, we are finding an arrangement of premise vectors utilizing lower arrange insights of the connections between the pixels. In particular, we expand the fluctuation between pixels to isolate straight conditions between pixels. ICA is a speculation of PCA in that it tries to recognize higharrange measurable connections between pixels to frame a superior arrangement of premise vectors. In a comparative design to PCA and LDA, once the new premise vectors are found, the preparation and test information are anticipated into the subspace and a strategy, for example, $\mathrm{NN}$ is utilized for grouping. The code for ICA was given by the creators to use in face acknowledgment look into. Free Component Analysis (ICA) is a measurable technique for changing a watched multidimensional irregular vector into its segments that are factually as autonomous from each different as conceivable [11]. ICA is a special case of redundancy reduction technique and it represents the data in terms of statistically independent variables.

\subsection{Scale Invariant Feature Transform (SIFT)}

SIFT proposed by Lowe solves the image rotation, affine transformations, intensity, and viewpoint change in matching features. The SIFT algorithm has 4 basic steps. First is to estimate scale-space extrema using the Difference of Gaussian (DoG). Secondly, a key point localization where the key point candidates are localized and refined by eliminating the low contrast points. Thirdly, a key point orientation assignment based on local image gradient and lastly a descriptor generator is to register the nearby picture descriptor for every key point in light of picture inclination size and orientation. The SIFT extraction process is based on the extraction of gradient samples from the image at the scale of the local interest point to describe. In its original formulation SIFT feature extraction was coupled with the scale-space representation of the DOG interest point detector. To increase the speed of the feature extraction process, the author used the pre-computed scalespace smoothed images to compute the SIFT descriptor feature. The SIFT descriptors are one of the most prominent local interest point descriptors. One of the main reasons for its success is its low complexity, which makes this detector fast and easy to implement. Another reason for the success of SIFT is the intrinsic invariance to small errors in the calculation of the position and area, resulting from representing the local image information with a histogram [12].

\subsection{Speeded-Up Robust Feature (SURF)}

SURF ("Speeded-Up Robust Features") approach has been designed as an efficient alternative to SIFT. SURF consolidates a Hessian-Laplace locale finder with a possess inclination introduction based component descriptor. Rather than depending on Gaussian subordinates for its inside calculations, it is however in view of straightforward $2 \mathrm{D}$ box channels ("Haar wavelets"). Those crate channels rough the impacts of the subordinate channel portions yet can be effectively assessed utilizing necessary pictures. Specifically, this assessment requires a similar consistent number of queries paying little respect to the picture scale, this expelling the requirement for the Gaussian pyramid. In spite of these rearrangements, SURF has been appeared to accomplish tantamount repeatability as finders on standard Gaussian subsidiaries, while yielding speedups of more than a variable of five contrasted with standard Difference of Gaussian. The 
SURF descriptor is likewise inspired by SIFT and seeks after a comparative spatial binning methodology, isolating the element locale into a $4 \times 4$ grid. The local image descriptor is meant to be as invariant as possible to variations in remaining image parameters, such as illumination and viewpoint. For feature description also SURF uses the wavelet responses. A neighborhood around the key point is selected and divided into sub regions and then for each subregion, the wavelet responses are taken and represented to get SURF feature descriptor. The sign of Laplacian which is already computed in the detection is used for underlying interest points. The sign of the Laplacian distinguishes bright blobs on dark backgrounds from the reverse case. In the case of matching, features are compared only if they have the same type of contrast (based on the sign) which allows faster matching [13].

\subsection{Oriented FAST and Rotated BRIEF (ORB)}

ORB (Oriented FAST and Rotated BRIEF) is a fast robust local feature detector, first presented in 2011 [14], that can be used in computer vision tasks like object recognition or 3D reconstruction. It is based on the visual descriptor BRIEF and the FAST key-point detector. Its aim is to provide a fast and efficient alternative to SIFT. The circle is fundamentally a combination of the FAST key-point finder and BRIEF descriptor with numerous adjustments to improve the execution. Sphere [14] expands on the outstanding FAST keypoint indicator and the as of late created BRIEF descriptor; consequently, we call it ORB (Oriented FAST and Rotated BRIEF). Both these strategies are appealing a result of their great execution and ease. Sphere incorporates,

* The expansion of a quick and precise introduction part to FAST.

* The effective calculation of arranged BRIEF components.

* Analysis of difference and relationship of situated BRIEF components.

* A learning technique for de-relating BRIEF components under rotational invariance, prompting to better execution in closest neighbor applications.

First, it utilizes FAST to discover key focuses, and after that apply Harris corner measure to discover best $\mathrm{N}$ focuses on them. It additionally utilizes the pyramid to deliver multiscale-highlights.

\section{LITERATURE REVIEW}

This paper examines the issue of face acknowledgment utilizing shading as an imperative sign in enhancing the exactness of acknowledgment. To perform acknowledgment of shading pictures, they utilize the attributes of a 3D shading sensor to create a shading LDA subspace, which thus can be utilized to perceive another test picture. To test the precision of our approach, we processed the acknowledgment rate crosswise over two shading face databases. Creators watch that the utilization of the LDA shading subspace altogether enhances acknowledgment exactness over the standard dark scale approach without giving up computational productivity [15].

A new system authored two-dimensional main segment investigation (2DPCA) is created for picture representation. Instead of PCA, 2DPCA depends on 2D picture grids as opposed to $1 \mathrm{D}$ vector so the picture lattice does not should be changed into a vector preceding element extraction. Rather, a picture covariance grid is built specifically utilizing the first picture frameworks and its eigenvectors are inferred for picture highlight extraction. To test 2DPCA and assess its execution, a progression of examinations were performed on three face picture databases: ORL, AR, and Yale confront databases. The acknowledgment rate over all trials was higher utilizing 2DPCA than PCA. The test comes about likewise showed that the extraction of picture elements is computationally more proficient utilizing 2DPCA than PCA [16].

Support vector machines (SVM) and independent component analysis (ICA) are two intense and moderately late strategies. SVMs are classifiers which have shown high speculation capabilities in many different tasks, including the object recognition problem. ICA is an element extraction method which can be viewed as a speculation of essential segment investigation (PCA). ICA has been mainly used to the problem of blind signal separation. In this paper, authors consolidate these two methods for the face acknowledgment issue. Analyses were made on two diverse face databases, accomplishing high acknowledgment rates. As the outcomes utilizing the mix PCA/SVM were not extremely distant from those got with ICA/SVM, these examinations propose that SVMs are generally heartless to the representation space. Thus as the training time for ICA is much larger than that of PCA, this result indicates that the best practical combination is PCA with SVM [17].

The Scale Invariant Feature Transform (SIFT) is a calculation used to distinguish and depict scale-, interpretation and turn invariant nearby components in pictures. The first SIFT calculation has been effectively connected by and large question location and acknowledgment errands, display sewing and others. One of its later uses likewise incorporates confront acknowledgment, where it was appeared to convey empowering comes about. To conquer the inadequacy of SIFT-based strategies, creators introduce novel face acknowledgment method that registers the SIFT descriptors at predefined (settled) areas learned amid the preparation organize. Thusly, it disposes of the requirement for key point location on the test pictures and renders our approach more powerful to enlightenment changes than related methodologies from the writing. Tests, performed on the Extended Yale B confront database, demonstrate that the proposed method contrasts positively and a few famous procedures from the writing as far as execution [18].

The Scale Invariant Feature Transform (SIFT) proposed by David G. Lowe has been utilized as a part of face acknowledgment and demonstrated to perform well. As of late, another indicator and descriptor, named Speed-Up Robust Features (SURF) recommended by Herbert Bay, draws in individuals' considerations. SURF is a scale and inplane pivot invariant indicator and descriptor with practically identical or shockingly better execution with SIFT. Since each of SURF highlight has just 64 measurements when all is said in done and an ordering plan is worked by utilizing the indication of the Laplacian, SURF is to a great extent than the 128-dimensional SIFT at the coordinating stride. In this manner in light of the above points of interest of SURF, writers propose to endeavor SURF includes in face acknowledgment in this article [12].

Feature coordinating is at the base of numerous PC vision issues, for example, protest acknowledgment or structure from motion. Current techniques depend on exorbitant descriptors 
for identification and coordinating. In this article, writers propose a quick parallel descriptor in view of BRIEF, called $\mathrm{ORB}$, which is revolution invariant and impervious to the commotion. Creators exhibit through analyses how ORB is at two requests of extent quicker than SIFT while executing too as a rule. The productivity is tried on a few genuine applications, including object discovery and fix following on a cell phone [14].

\section{COMPARATIVE TABLE}

In this paper, we surveyed and compare various face recognition method which was used to find out the performance of numerous algorithms. Using this system selection of the algorithms we compare the performance of the recognition rate with one other technique was generated for all the algorithms separately. This information was used to plot the graphs comparing the above-mentioned rate performances of the six algorithms under study. Comparative table and graph are shown below: -

Table 1. Comparative Study

\begin{tabular}{|c|c|c|c|}
\hline S. No. & Approaches & Merits & Demerits \\
\hline 1. & PCA & $\begin{array}{l}\text { a. Low Noise Sensitivity } \\
\text { b. It decreased requirements for capacity and } \\
\text { memory. } \\
\text { c. Increased efficiency gave the processes taking } \\
\text { place in smaller dimensions }\end{array}$ & $\begin{array}{l}\text { a. Reduced complexity in images' grouping } \\
\text { with the use of PCA } \\
\text { b. Reduction of noise since the maximum } \\
\text { variation basis is chosen and so the small } \\
\text { variations in the background are ignored } \\
\text { automatically } \\
\text { c. Lack of redundancy of data given the } \\
\text { orthogonal components }\end{array}$ \\
\hline 2. & ICA & $\begin{array}{l}\text { a. It provides a better probabilistic model of the } \\
\text { data, which better identifies where the data } \\
\text { concentrate in } n \text {-dimensional space } \\
\text { b. It finds a not necessarily orthogonal basis } \\
\text { which may reconstruct the data better than } \\
\text { PCA in the presence of noise } \\
\text { c. It is sensitive to high-order statistics in the } \\
\text { data, not just the covariance matrix }\end{array}$ & $\begin{array}{l}\text { a. The covariance matrix is difficult to be } \\
\text { evaluated in an accurate manner } \\
\text { b. Even the simplest invariance could not be } \\
\text { captured by the ICA unless the training } \\
\text { data explicitly provide this information }\end{array}$ \\
\hline 3. & LDA & $\begin{array}{l}\text { a. Linear discriminant group images of the same } \\
\text { class and separates images of different classes } \\
\text { of the images } \\
\text { b. LDA is processed fast compared to PCA in } \\
\text { some cases } \\
\text { c. LDA has small Error rate }\end{array}$ & $\begin{array}{l}\text { a. The dimension of all images should be } \\
\text { same. } \\
\text { b. Works on only small database } \\
\text { c. The face to classify must be in database }\end{array}$ \\
\hline 4. & SIFT & $\begin{array}{l}\text { a. SIFT is appropriate to check images having } \\
\text { nearly same illumination, with a different } \\
\text { posture, expression, accessories etc. } \\
\text { b. The key points of objects are first extracted } \\
\text { from a set of reference images as well as from } \\
\text { the test image }\end{array}$ & $\begin{array}{l}\text { a. Quite complex and not as accurate as } \\
\text { required by the police information system } \\
\text { b. SIFT reducing the quality of the detected } \\
\text { points }\end{array}$ \\
\hline 5. & SURF & $\begin{array}{l}\text { a. SURF is much faster than the 128-dimensional } \\
\text { SIFT at the matching step. } \\
\text { b. SURF features are more robust to the change } \\
\text { of the ratio threshold }\end{array}$ & $\begin{array}{l}\text { a. Quite complex and not as accurate as } \\
\text { required by the police information system } \\
\text { b. Need to improvement in matching speed. }\end{array}$ \\
\hline 6. & ORB & $\begin{array}{l}\text { a. ORB is robust to noise and affine } \\
\text { transformation. } \\
\text { b. ORB uses binary test between the pixels, } \\
\text { which is much faster than other feature } \\
\text { descriptor algorithms. } \\
\text { c. The Keypoint detection in ORB is much faster } \\
\text { than SURF, this is due to the fact that it } \\
\text { doesn't use orientation component, unlike } \\
\text { SURF which has computation overhead. }\end{array}$ & $\begin{array}{l}\text { a. ORB takes more Time to process face data } \\
\text { set. }\end{array}$ \\
\hline
\end{tabular}




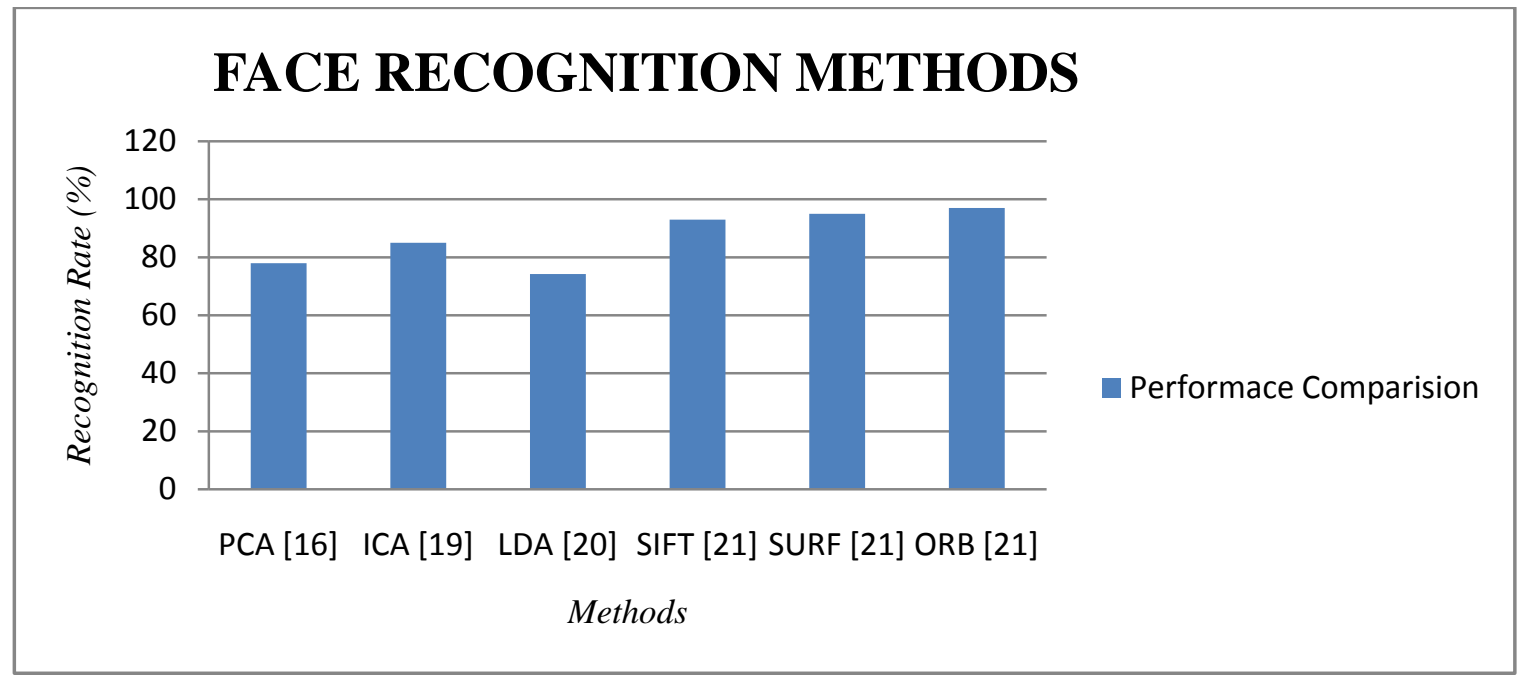

Figure 2: Performance on Different Approaches

Face recognition in computer vision is a very most frequent application that prevents unauthorized users and gets access features of the legitimate user. Using graph, we have analyzed that these approaches have shortcoming and benefit separately. Here we compare methods performance which has already implemented for better recognition and detection with their tremendous recognition face data rate. Also, we listed some of merits and demerits of these techniques that ensure which one is best for face recognition. But there is no complete solution for identifying accurate recognition; still, we concluded using survey of method ORB is better than all approaches.

\section{CONCLUSION}

In this paper, we tried to throw some light on some of the states of art techniques and some recent techniques trying to overcome the shortcomings of the contemporary techniques. We mainly focused on the appearance and feature-based approaches to face recognition. We presented a comparative study of frequently used most popular face recognition method (PCA, LDA, ICA, SIFT, SURF, ORB). It was found that no algorithm-metric combination is the state-of-the-art at this time, and the space of algorithm comparisons needs further research. But in this survey paper, we analyzed that feature-based image matching algorithm SURF, SIFT, and ORB of face detection and feature extraction among this ORB are much proficient in delivering the best result for face recognition. While we use this $\mathrm{ORB}$, to combine with particle swarm optimization (PSO), make hybrid approach which best and suitable for features with optimized form. That hybrid approach is utilized for identifying multiple face objects in the given image.

\section{REFERENCES}

[1] Kumar, Sanjeev, and Harpreet Kaur. "Face recognition techniques: Classification and comparisons." International Journal of Information Technology and Knowledge Management 5, no. 2 (2012): 361-363

[2] Ramadan, Rabab M., and Rehab F. Abdel-Kader. "Face recognition using particle swarm optimization-based selected features." International Journal of Signal Processing, Image Processing and Pattern Recognition 2, no. 2 (2009): 51-65.
[3] Vijayakumari, V. "Face recognition techniques: A survey." World Journal of Computer Application and Technology 1, no. 2 (2013): 41-50.

[4] Besl, Paul J., and Neil D. McKay. "Method for registration of 3-D shapes." In Robotics-DL tentative, pp. 586-606. International Society for Optics and Photonics, 1992.

[5] Turk, M. "A. Pentland Eigenfaces for Recognition Journal of Cognitive Neuroscience, Volume 3, Number 1." (1991).

[6] Harguess, Josh, and J. K. Aggarwal. "A case for the average-half-face in 2D and 3D for face recognition." In 2009 IEEE Computer Society Conference on Computer Vision and Pattern Recognition Workshops, pp. 7-12. IEEE, 2009.

[7] Phillips, P. Jonathon, Harry Wechsler, Jeffery Huang, and Patrick J. Rauss. "The FERET database and evaluation procedure for face-recognition algorithms." Image and vision computing 16, no. 5 (1998): 295-306.

[8] Krzanowski, W. J., Philip Jonathan, W. V. McCarthy, and M. R. Thomas. "Discriminant analysis with singular covariance matrices: methods and applications to spectroscopic data." Applied statistics (1995): 101-115.

[9] Mandal, B., X. D. Jiang, and A. Kot. "Multi-scale feature extraction for face recognition." In Industrial Electronics and Applications, 2006 1ST IEEE Conference on, pp. 16. IEEE, 2006.

[10] Bartlett, Marian Stewart, Javier R. Movellan, and Terrence J. Sejnowski. "Face recognition by independent component analysis." IEEE Transactions on neural networks 13, no. 6 (2002): 1450-1464.

[11] Hyvarinen, Aapo. "Fast and robust fixed-point algorithms for independent component analysis." IEEE transactions on Neural Networks 10, no. 3 (1999): 626634.

[12] Lowe, David G. "Distinctive image features from scaleinvariant keypoints." International journal of computer vision 60, no. 2 (2004): 91-110.

[13] Bay, Herbert, Andreas Ess, Tinne Tuytelaars, and Luc Van Gool. "Speeded-up robust features 
(SURF)." Computer vision and image understanding 110, no. 3 (2008): 346-359.

[14] Rublee, Ethan, Vincent Rabaud, Kurt Konolige, and Gary Bradski. "ORB: An efficient alternative to SIFT or SURF." In 2011 International conference on computer vision, pp. 2564-2571. IEEE, 2011.

[15] Thomas, Mani, Chandra Kambhamettu, and Senthil Kumar. "Face recognition using a color subspace LDA approach." In 2008 20th IEEE International Conference on Tools with Artificial Intelligence, vol. 1, pp. 231-235. IEEE, 2008.

[16] Yang, Jian, David Zhang, Alejandro F. Frangi, and Jingyu Yang. "Two-dimensional PCA: a new approach to appearance-based face representation and recognition." IEEE transactions on pattern analysis and machine intelligence 26, no. 1 (2004): 131-137.

[17] Déniz, Oscar, M. Castrillon, and Mario Hernández. "Face recognition using independent component analysis and support vector machines." Pattern recognition letters 24 , no. 13 (2003): 2153-2157.
[18] Križaj, Janez, Vitomir Štruc, and Nikola Pavešić. "Adaptation of SIFT features for robust face recognition." In International Conference Image Analysis and Recognition, pp. 394-404. Springer Berlin Heidelberg, 2010.

[19] Yuen, Pong C., and Jian-Huang Lai. "Face representation using independent component analysis." Pattern recognition 35, no. 6 (2002): 1247-1257.

[20] Fernandes, Steven, and Josemin Bala. "Performance Analysis of PCA-based and LDA-based Algorithms for Face Recognition." International Journal of Signal Processing Systems 1, no. 1 (2013): 1-6.

[21] Karami, Ebrahim, Siva Prasad, and Mohamed Shehata. "Image Matching Using SIFT, SURF, BRIEF and ORB: Performance Comparison for Distorted Images."

[22] Shukla, Shivang, and Sourabh Dave. "Comparison of Face Recognition algorithms \& its subsequent impact on side face." In ICT in Business Industry \& Government (ICTBIG), International Conference on, pp. 1-8. IEEE, 2016. 